2. G. Hermann, Die Frage der endlich vielen Schritte in der Theorie der Polynomideale, Math. Ann. 95 (1925), 736-788.

3. E. Noether, Eliminationstheorie und allgemeine Idealtheorie, Math. Ann. 90 (1923), 229-261.

4. J.-P. Serre, Corps locaux, Hermann, Paris, 1962.

5. B. L. Van der Waerden, Modern algebra. Vols. I, II, 2nd ed., Ungar, New York, 1937-1940.

Institut des Hautes Etudes Scientifiques, France and Brown University

\title{
VARIETIES OF GROUPS AND BURNSIDE'S PROBLEM
}

\author{
BY L. G. KOVÁCS
}

Communicated by Michio Suzuki, December 15, 1967

If $\mathfrak{B}$ is a variety of groups and $d$ is a positive integer, $\mathfrak{B}^{(d)}$ denotes the variety consisting of the groups whose $d$-generator subgroups are all in $\mathfrak{B}$. In a recent paper [3], B. H. Neumann formulated the Extended Burnside Problem:

Problem 7 . Let $\mathfrak{B}$ be a locally finite variety and $d \geqq 1$ an integer. Is $\mathfrak{B}^{(d)}$ locally finite?

He went on to ask two related questions:

Problem 8 . Is there, to each locally finite variety $\mathfrak{B}$, an integer $d=d(\mathfrak{B})$ such that $\mathfrak{B}^{(d)}$ is locally finite?

Problem 9. Do the locally finite groups in $\mathfrak{B}^{(d)}$, where $\mathfrak{B}$ is a locally finite variety, form a variety?

Neumann called the latter the Restricted Extended Burnside Problem. One might derive from it, like Problem 8 from Problem 7, the following:

Problem N. Is there, to each locally finite variety $\mathfrak{B}$, an integer $n=n(\mathfrak{B})$ such that the locally finite groups in $\mathfrak{B}^{(n)}$ form a variety?

The purpose of this note is to present reduction theorems for Problem 8 and Problem N, similar to the Hall-Higman reduction theorems [1] for the classical forms of Burnside's Problem.

THEOREM 1. If $\mathfrak{B}$ is a locally finite and locally soluble variety, $\mathfrak{B}_{L N}$ is the variety consisting of the locally nilpotent groups of $\mathfrak{B}$, and $\mathfrak{B}_{L N}{ }^{(a)}$ is locally finite for some integer $d$, then $\mathfrak{B}^{\left(d^{*}\right)}$ is locally finite for some integer $d^{*}$.

This is a direct consequence of (c) of the forthcoming paper [2]. Theorem 2 will be derived from the following part of (b) of [2]: 
LEMma. If $\mathfrak{U}$ is a locally finite variety which contains only finitely many (isomorphism classes of) finite simple groups, and if $\mathfrak{W}_{1}$ is the class of those groups whose nilpotent factors and simple factors all belong to $\mathfrak{U}$, then $\mathfrak{B}_{1}$ is a locally finite variety.

In order to formulate Theorem 2 in full generality, some additional terminology is required. According to M. B. Powell (cf. Sheila Oates [4]), the complexity of a finite simple group $S$ is 0 if $S$ is abelian, and $k+1$ if $S$ is not abelian and $k$ is the largest complexity which occurs for the proper simple factors of $S$. In particular, the groups of complexity 1 are precisely the minimal simple groups of J. G. Thompson [5]. Attempts of Oates and Powell (cf. [4]) have raised the hope that the near future may bring a proof of the

CONJECTURE. For each nonnegative integer $k$, there exists an integer $m(k)$, depending on $k$, such that:

(k) Every finite simple group of complexity at most $k$ can be generated by $m(k)$ elements.

Obviously, (0) is valid with $m(0)=1$, and Thompson's classification of the minimal simple groups [5] implies (1) with $m(1)=2$. It has, of course, been long conjectured that $(\mathrm{k})$ is always true with $m(k)=2$; but no proof of this stronger claim is within sight, and for the present context it does not matter how generous one is in overestimating $m(k)$.

THEOREM 2. Let $\mathfrak{B}$ be a locally finite variety such that the locally finite groups in $\mathfrak{B}_{L N}^{(a)}$ form a variety $\mathfrak{H}_{1}$ and the finite simple groups in $\mathfrak{B}$ all have complexity less than $k$. If $(\mathrm{k})$ is valid, then the locally finite groups in $\mathfrak{B}^{\left(d^{*}\right)}$ form a variety where $d^{*}=\max \{2, d, m(k)\}$. In particular, if $\mathfrak{B}$ is locally soluble (so that $k$ can be chosen as 1 ), then the locally finite groups in $\mathfrak{B}^{\left(d^{*}\right)}$ form a variety where $d^{*}=\max \{2, d\}$.

Proof. As $\mathfrak{B}_{L N}^{\left(d^{*}\right)}$ is obviously contained in $\mathfrak{B}_{L N}^{(d)}$, the finite nilpotent groups of $\mathfrak{B}^{\left(d^{*}\right)}$ all lie in $\mathfrak{U}_{1}$. Let $S$ be a finite simple group in $\mathfrak{B}^{\left(d^{*}\right)}$. If $S$ can be generated by $d^{*}$ elements, then it lies in $\mathfrak{B}$, has complexity less than $k$, and the order of $S$ is at most that of the $\mathfrak{B}$-free group of rank $d^{*}$. If $S$ cannot be generated by $d^{*}$ elements, then (k) implies that the complexity of $S$ is greater than $k$. Thus $\mathfrak{B}^{\left(d^{*}\right)}$ has no groups of complexity precisely $k$, hence it cannot contain groups of complexity greater than $k$ either, and it follows that $\mathfrak{B}^{\left(d^{*}\right)}$ contains only finitely many (isomorphism classes of) finite simple groups. Let $\mathfrak{U}$ be the variety generated by $\mathfrak{U}_{1}$ together with these simple groups: then $\mathfrak{U}$ is locally finite and, as $\mathfrak{U}_{1}$ is also locally nilpotent, $\mathfrak{U}$ contains only finitely many finite simple groups. (Use, for instance, (4) of [2].) Let $\mathfrak{B}_{1}$ be as in the Lemma. Then the finite groups of $\mathfrak{B}^{\left(d^{*}\right)}$ are all in 
$\mathfrak{W}_{1}$, and hence so are all the locally finite groups of $\mathfrak{B}^{\left(d^{*}\right)}$. Thus the class of the locally finite groups of $\mathfrak{B}^{\left(d^{*}\right)}$ is precisely the variety $\mathfrak{B}_{1} \cap \mathfrak{B}^{\left(d^{*}\right)}$.

\section{REFERENCES}

1. P. Hall and Graham Higman, On the p-length of p-soluble groups and reduction theorems for Burnside's Problem, Proc. London Math. Soc. (3) 6 (1956), 1-42.

2. L. G. Kovács, Varieties and finite groups, J. Austral. Math. Soc. (to appear).

3. B. H. Neumann, Varieties of groups, Bull. Amer. Math. Soc. 73 (1967), 603613.

4. Sheila Oates, Identical relations in a small number of variables, Proc. Internat. Conf. Theory of Groups, Austral. Nat. Univ. Canberra, August 1965, Gordon and Breach, New York, 1967, pp. 261-264.

5. John G. Thompson, Nonsolvable finite groups whose nonidentity solvable subgroups have solvable normalizers (to appear).

Australian National University, Canberra 